\title{
ASA CLASSIFICATION IN PEDIATRIC ANESTHESIA
}

Natalie T. Buu MD, J. Mark Ansermino MBB Ch

Department of Pediatric Anesthesia, B.C. Children's Hospital, 4480 Oak Street, Vancouver, BC V6H 3V4

INTRODUCTION: ASA classification was introduced in 1941(1) by the American Society of Anesthetists. Designed for the adult patient, it was never intended to represent peri-operative risk of morbidity, yet, it is often used in surgical and anesthetic research as an indication of peri-operative risk (2). This survey aimed to assess the consistency of ASA physical status classification as allocated by Canadian pediatric anesthesiologists.

METHODS: After ethical approval was obtained, emails were sent to all 228 members of the Pediatric Section of the Canadian Anesthesiologists' Society inviting them to participate in a web-based survey that presented ten hypothetical pediatric patients in need of surgery. Respondents were asked to allot an ASA class to each patient. Two reminders were sent. Responders were also asked about their level of experience in anesthesia, percentage of pediatric anesthesia in their practice and their province of work. The survey was created using Quask ${ }^{\circledR}$, data maintained in Excel databases, and results analyzed using Quask ${ }^{\circledR}$ and Excel $^{\circledR}$.

RESULTS: 15 of the 228 initial emails were returned as undeliverable. 7 members declined to participate. Completed questionnaires were obtained from 117 of the 206 remaining members $(56.8 \%)$. In no case was there unanimous assignment of a particular ASA physical status classification, though in one case, allocations were between 2 possible grades. In five others, responses ranged among 3 grades and in four cases responses ranged among 4 grades. Nine out of ten provinces were represented and $75.2 \%$ of respondents had more than 5 years of anesthesia experience. $68.4 \%$ of respondents worked in institutions which deal solely in pediatric anesthesia.

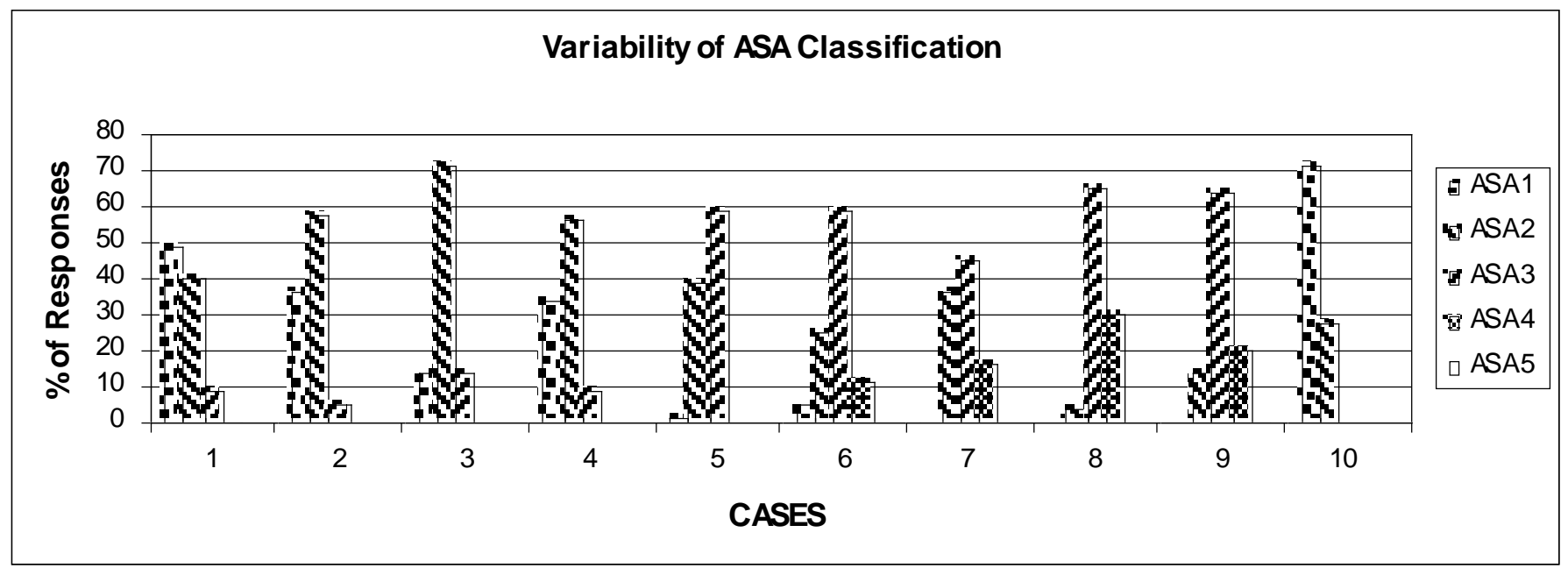

DISCUSSION: The variability in ASA classification is similar in this study to comparable studies done in adults $(3,4)$. It cannot reliably be used alone to describe a patient's physical status. Certain aspects of patient histories in children may render ASA allocation more challenging. These findings may suggest the need for an alternative system, more clinically relevant to the pediatric population, and more applicable for future studies in anesthesia and surgical research.

\section{REFERENCES:}

1. An sth sio b g y2:281-284.

2. Br. J. An esth. 77: 217-22.

3. An eth sia 50:195-199.

4. An esth .In en sive Care 30:633-640. 